\title{
Optimal Mix of Wind Generation and Energy Storage Systems in Power Distribution Networks
}

\author{
A. D. Petropoulos ${ }^{\mathrm{a}}$, N. C. Koutsoukis ${ }^{\mathrm{a}}$, E. S. Karapidakis ${ }^{\mathrm{b}}$, Member, IEEE, P. S. Georgilakis ${ }^{\mathrm{a}}$, Senior Member, IEEE \\ ${ }^{a}$ School of Electrical and Computer Engineering, National Technical University of Athens (NTUA), GR 15780, Athens, Greece, \\ e-mail: pgeorg@power.ece.ntua.gr \\ ${ }^{\mathrm{b}}$ Department of Natural Resources and Environment, Technological Educational Institute of Crete, GR 73133, Chania, Greece, \\ e-mail: karapidakis@chania.teicrete.gr
}

\begin{abstract}
The need for high penetration of renewable energy sources poses several economic and technical challenges in distribution system planning and operation. In order to confront effectively these challenges, the integration of energy storage systems (ESSs) is proposed. More specifically, in this paper, an optimal power flow (OPF) based technique is developed for the optimal sizing of wind generation units and ESSs as well as their optimal operation strategy, considering the ESSs' reactive power contribution and a curtailment factor in wind generation. Application results on a 34-bus distribution network illustrate the performance of the proposed method.
\end{abstract}

Index Terms - Active-reactive optimal power flow, distributed generation units, wind energy, energy storage, reactive power capability

\section{INTRODUCTION}

$\mathrm{E}_{\mathrm{p}}^{\mathrm{n}}$ nviromental objectives and the promotion of sustainable power systems are the major drivers in the integration of renewable energy sources (RES).However, the implementation of distributed generation (DG) units in the electrical grids poses multiple technical and economic issues [1]. The investment costs of wind and solar renewable energy sources are still higher compared with the conventional units. As a result, there are certain governmental legislations to provide incentives for RES' implementation in the power system [2]. Furthermore, the achievement of the EU climate and energy package [3] formulates an environment in which RES are very competitive in comparison with conventional sources. As a result, the penetration of renewable energy in the power grids is expected to increase in the near future [4].

The stochastic behavior of non-dispatchable renewable energy sources (e.g. wind and solar) in combination with their increasing penetration in distribution networks may have negative effects in the entire grid. A solution is the integration of Energy Storage Systems (ESSs), which can help minimize the deviations between the load and the generated power from RES and ESS. In addition, the integration of ESSs in distribution networks with non-dispatchable renewable distributed generation can benefit the power quality and the reliability of the overall power distribution system, even if ESSs' cost is still remarkable [1].

Among the benefits of ESSs is their capability to generate both active and reactive power through power conditioning systems (PCSs). This capability should be fully utilized so as to compensate the PCSs' high installation cost and is the basis of the simultaneous optimization of active and reactive strategies in distribution networks [6].

However, the benefits and costs of the ESSs should be carefully evaluated considering the details of the problem at hand. In [7], an optimal power flow (OPF) based method was employed for the optimal citing and sizing of ESSs and a cost/benefit analysis was suggested to investigate the possibility of their installation. A combined active-reactive optimal power flow (A-R OPF) method is proposed in [6], which leads to a remarkable power loss reduction and to a reduction of the reactive energy imported from the transmission network considering the contribution of ESSs' reactive power.

In this paper, an OPF-based method is proposed for the optimal sizing of wind-based DG units and ESSs as well as their optimal operation strategy in order to maximize the revenue of the distribution network operator, who is assumed to be also the owner of the DG units. Initially, the incorporation of a generation-load model within an OPFtechnique is proposed for the optimal mix of wind DG units and ESSs. After the optimal mix calculation, the ESSs' reactive power capability is investigated. At this stage, considering the ESSs' reactive power contribution along with a curtailment index for wind DG output power, an A-R OPF method is proposed in order to define the ESSs' and DG units' optimal operation strategy.

These two optimization problems are formulated as nonlinear programming (NLP) problems that are solved using the General Algebraic Modeling System (GAMS) environment [8]. A 34-bus distribution network is used for the evaluation of the proposed method.

The paper is organized as follows: Section II offers a comprehensive review of the benefits of ESSs. The problem formulation is presented in Section III. In Section IV, the proposed method is applied in a 34-bus distribution system and the potential of various scenarios and their impact on grid performance are discussed. Conclusions are drawn in Section $\mathrm{V}$. 


\section{BENEFITS OF ESS}

\section{A. Financial Benefits}

Electricity price varies according to the energy demand from the consumers. This concept is usually used by utilities for formulating different tariffs during low and high demand [9]. Generally, there are countries like Canada in which a 24 different hourly prices model is applied. Although, based on the time-of-use pricing, a simpler tariff model (two to three price periods) can provide a good fit to hourly prices model [10]. In this study, a two tariff model is selected.

The energy storage systems exploit the charging policy (price differs during time periods) in order to arise financial benefits. For instance, when the daily demand is low (off-peak period), a low electricity price is formed, while a high electricity price corresponds to the high demand (on-peak) period.

Generally, the daily demand is lower than wind power during certain intervals (off-peak period). During these intervals, there is an excess of wind power which is able to be stored in the ESSs. This stored energy can be consumed during the time period in which the demand is higher than wind power (on-peak period) and the electricity price is high. Thus, the stored energy is consumed during high price period to cover the high demand. At this period, ESSs exploit the advantage of tariff difference between on-peak and off-peak price and the financial benefits from their usage are maximized.

\section{B. Technical Benefits}

A PCS has the capability of generating both active and reactive power in all four quadrants [11]. Thus, the main advantages of the ESSs integration in a power system are:

○ The reduction of energy losses;

- The improvement of power system's bus voltages profile;

- The reduction of imported reactive energy from the transmission network.

Generally, all the above mentioned positive effects are illustrated by the case study presented in Section IV. For instance, a PCS is capable to dispatch or absorb reactive power in order to improve electric grid's characteristics without violating the problem constraints.

\section{PROBLEM FoRMULATION}

This section formulates the two optimization problems, namely the planning and the operation problems.

\section{A. Planning Problem}

The planning problem accommodates a generation-load model into a deterministic optimal power flow analysis in order to maximize the total yield from the wind energy and the energy supplied from the ESSs, taking into account all the technical constraints of the distribution network.

More specifically, for each system state the power flow equations (4), (5) are solved and the generation of the wind DG units and ESSs are computed based on the energy demand of each state. Each state has 24 hourly values. Various representative states are used to model the annual load curve, where each state has its own duration. The optimum size of the wind DG units and the ESSs is then determined so that for all the operating conditions (system states), the penetration of DG and storage units in distribution system is maximized without violating the power system constraints.

The planning problem leads to a non-linear programming (NLP) problem, which consists of three design variables, the rated power of wind DG units $\left(P_{\text {wdg }}\right)$, the installed ESS's capacity $\left(E_{e s s}\right)$ and the upper bound of apparent power $\left(S_{\max }\right)$ of ESS's power conditioning system (PCS). In this paper, the charge rate $\left(S_{\max } / E_{\text {ess }}\right)$, which has unit $1 / \mathrm{h}$, equals to 0.5 . Moreover, it is considered that the ESS's power factor is unity, thus ESSs yield only active power. The computed optimal values of the design variables $\left(P_{w d g}, E_{e s s}\right.$ and $\left.S_{\max }\right)$ are continuous, which are then rounded to the closest discrete values, taking into account the available sizes from the manufacturers' data sheets [12], [13].

To obtain a reasonably accurate planning strategy that determines the optimal mix of wind DG units and ESSs, the following assumptions are made:

1. Fourteen (14) representative power system states (each composed of 24 hours) are considered. More specifically, (a) three typical days (peak, average and minimum) for each one of the four seasons, (b) one day with peak load and minimum wind speed, and (c) one day with minimum load and peak wind speed.

2. The hourly wind speed data and load demand were the same as the ones used in [14].

3. All wind DG units are operating at 0.9 lagging power factor.

4. There will be no system upgrade, such as changing feeder capacity.

5. A two tariff electricity price model is considered, in which the electricity price is high when the demand is high, while the electricity price is low when the demand is low.

6. There is an investment cost limit.

The objective function of the planning problem is as follows:

$$
\max \left\{F_{1}-F_{2}\right\}
$$

where $F_{1}$ is the total annual income from the energy produced by the wind DG and the ESSs and $F_{2}$ is the annual cost of energy losses:

$$
F_{l}=\sum_{t=1}^{T} C_{p r}(t) \sum_{i=1}^{N}\left(P_{w d g}(i, t)+P_{d i s}(i, t)-P_{c h}(i, t)\right)
$$

where $T$ is the time intervals (14 days / 336 hours), $C_{p r}(t)$ is the electricity price during hour $\mathrm{t}, N$ is the total number of buses in the system, $P_{w d g}(i, t)$ is the output power of wind DG unit at bus $i$ during hour $t, P_{c h}(i, t)$ and $P_{d i s}(i, t)$ is the active power charge and discharge of ESS at bus $i$ during hour $t$, 
respectively.

The annual cost of energy losses is computed by:

$$
F_{2}=\sum_{t=1}^{T} C_{p r}(t) \cdot P_{\text {losses }}(t)
$$

where $P_{\text {losses }}(t)$ is the system's power losses during hour $t$.

The optimization of (1) is subject to constraints (4)-(14). 1) Power flow constraints

$$
\begin{aligned}
& P_{S S}(t)+P_{w d g}(t)+P_{d i s}(t)-P_{c h}(t)-P_{d}(t)= \\
& \sum_{j=1}^{N} V_{i}(t) \cdot V_{j}(t) \cdot Y_{i j} \cdot \cos \left(\theta_{i j}(t)+\delta_{j}(t)-\delta_{i}(t)\right) \\
& Q_{S S}(t)+Q_{w d g}(t)-Q_{d}(t)= \\
& \sum_{j=1}^{N} V_{i}(t) \cdot V_{j}(t) \cdot Y_{i j} \cdot \sin \left(\theta_{i j}(t)+\delta_{j}(t)-\delta_{i}(t)\right)
\end{aligned}
$$

where $P_{S S}(t)$ is the substation active power injected at slack bus at hour $t, P_{d}(t)$ is the active power demand at hour $t$, $Q_{S S}(t)$ is the substation reactive power injected at slack bus during hour $t$, and $Q_{d}(t)$ is the reactive power demand at hour $t$.

2) Voltage limits

$$
0.94 \text { p.u. } \leq V(i, t) \leq 1.06 \text { p.u. } \quad \forall \mathrm{i} \in N
$$

3) Feeder capacity limits

$$
S(i, j, t) \leq S_{\max }(i, j) \quad \forall i, j \in N
$$

4) Power bounds at slack bus

$$
\begin{aligned}
& -P_{\text {reverse }} \leq P_{S S}(t) \leq P_{\text {forward }} \\
& -Q_{\text {reverse }} \leq Q_{S S}(t) \leq Q_{\text {forward }}
\end{aligned}
$$

These constraints define that the reverse power flow at the slack bus will not exceed $60 \%$ of the substation rating.

5) Wind penetration limits

$$
x_{\min } \sum_{i=1}^{N} P_{\text {d.peak }}(i) \leq \sum_{i=1}^{N} P_{w d g}(i) \leq x_{\max } \sum_{i=1}^{N} P_{d . p e a k}(i)
$$

where $x_{\min }$ and $x_{\max }$ are the lower and upper limits of wind power penetration.

6) ESS's equations

The energy storage level $E(i, t)$ in a storage unit at bus $i$ during hour $t$ is computed by:

$$
E(i, t)=E(i, t-1)+n_{c h} \cdot P_{c h}(i, t)-\frac{P_{d i s}(i, t)}{n_{d i s}}
$$

where $n_{c h}$ and $n_{d i s}$ are the charge and discharge efficiency, respectively. In order to prolong the ESS lifetime, the following constraint is applied:

$$
20 \% \cdot E_{e s s}(i) \leq E(i, t) \leq 90 \% \cdot E_{e s s}(i)
$$

Generally, ESS will be charged mainly at night (when wind power is higher than power demand / off-peak period) and discharge during the day (when wind power is lower than demand / on-peak period). So, one charge/discharge cycle per day is considered. If ESSs are able to store more energy, than the one provided by the wind DG units, then the extra energy will be provided by the transmission system, through the substation. Furthermore, it is assumed that the reverse power flow is obligatory to come from the wind generators. As a result, the output power of ESSs must be less than the total demand for every time interval. A construction constraint of PCS is related to maximum charging and discharging power, $S_{\max }$, during that hour:

$$
\begin{gathered}
P_{c h}(i, t) \leq S_{\text {max }}(i) \\
P_{d i s}(i, t) \leq S_{\text {max }}(i)
\end{gathered}
$$

\section{B. Operation Problem}

This optimization problem deals with the optimal operation strategy of the DG units and ESSs that were optimally sized according to the planning problem of Section II.A. For the optimization of the operation problem a curtailment index is introduced, which has values between zero and one. This index guarantees that during high wind speed there will be no violation of the system's constraints. Moreover, the ESSs' reactive power contribution is taken into account.

An Active - Reactive OPF (21), (22) is implemented in order to estimate the effect of the ESSs' reactive power contribution to energy losses of the network, the total incoming reactive energy from the transmission network and the profile of the power system's voltages.

The optimization of the operation strategy is also a nonlinear programming problem, which consists of four design variables, the curtailment factor of wind output power ( $C T)$, the dispatched reactive power of the ESS ( $Q_{\text {disp }}$ ), the active power charge of the ESS $\left(P_{c h}\right)$ and the active power discharge of the ESS ( $\left.P_{\text {disch }}\right)$.

An ESS is considered in this paper to consist of a PCS unit and a storage unit. A PCS unit is a 4-quadrant inverter which independently controls the real power $(\mathrm{P})$ and reactive power (Q). Providing independent control of the real and reactive power allows the system operator to use the reactive capacity of the PCS during times where the real power demand is low, providing voltage support or power factor compensation. So the ESS's capability of reactive power does not require storage unit to be present. For instance, PCS is able to provide reactive power by altering its phase angle so as to influence the network phase angle, thus making the power converter behave 
either as a capacitor or as an inductor. However if active power support is required then ESSs can use the stored energy to supply power.

Finally, the independent values for P, Q and S power limits can be programmed into the PCS. As a result, there should be a compromise between the reactive power $\left(Q_{a v a}\right)$ and the active power of a PCS. The first quantity relates with the cost of energy losses and the other with the total energy yield. The capability of PCS is limited between $\pm S_{\max }$. So if the power converter absorbs active power $P$, the remaining part until $S_{\max }$ can be used for reactive power (absorb or supply $Q_{a v a}$ ):

$$
Q_{a v a}(i, t)=\left\{\begin{array}{l} 
\pm \sqrt{S_{\max }^{2}(i)-P_{c h}^{2}(i, t)}, t \in \text { off - peak period } \\
\pm \sqrt{S_{\max }^{2}(i)-P_{d i s}^{2}(i, t)}, t \in \text { on - peak period }
\end{array}\right.
$$

Equation (15) defines the available amount of reactive power that can be utilized. However, not all of this amount will be utilized because of various reasons, such as electricity prices, and only a proportion $\left(Q_{\text {disp }}\right)$ of it will be utilized.

According to power capability curve of the PCS, $Q_{\text {disp }}$ can be calculated as follows:

$$
\begin{gathered}
P_{\text {dis }}^{2}(i, t)+Q_{\text {disp }}^{2}(i, t) \leq S_{\max }^{2}(i) \\
P_{c h}^{2}(i, t)+Q_{\text {disp }}^{2}(i, t) \leq S_{\max }^{2}(i) \\
Q_{\text {disp }}(i, t) \geq-S_{\max }(i)
\end{gathered}
$$

Due to the curtailment factor, the objective function of the operation problem is given by (19), which is different in comparison with the objective (1) of the planning problem:

$$
\max \left\{F_{3}-F_{2}\right\}
$$

where,

$$
F_{3}=\sum_{t=1}^{T} C_{p r}(t) \sum_{i=1}^{N}\left(P_{w d g}(i, t) \cdot C T(i, t)+P_{d i s}(i, t)-P_{c h}(i, t)\right)
$$

The optimization of (19) is subject to constraints (6)-(14) together with the modified power flow equations (21) and (22):

$$
\begin{gathered}
P_{S S}(t)+P_{w d g}(t) \cdot C T(t)+P_{d i s}(t)-P_{c h}(t)-P_{d}(t)= \\
\sum_{j=1}^{N} V_{i}(t) \cdot V_{j}(t) \cdot Y_{i j} \cdot \cos \left(\theta_{i j}(t)+\delta_{j}(t)-\delta_{i}(t)\right) \\
Q_{S S}(t)+Q_{w d g}(t)+Q_{d i s p}(t)-Q_{d}(t)= \\
\sum_{j=1}^{N} V_{i}(t) \cdot V_{j}(t) \cdot Y_{i j} \cdot \sin \left(\theta_{i j}(t)+\delta_{j}(t)-\delta_{i}(t)\right)
\end{gathered}
$$

\section{RESULTS AND DISCUSSION}

The planning and the operation problems of Section III were implemented in GAMS environment and solved using the CONOPT3 NLP solver. The system under study is a 34-bus distribution network with a peak load of $4.635+2.8735 j$ MVA. The detailed data of the network under study can be found in [15]. The candidate buses for installing the DG units are in the set B1: $\{5,14,21,31\}$ and for installing the ESSs are in the set B2: $\{3,19,31\}$. The electricity price during the on-peak and off-peak period is assumed to be $0.74 € / \mathrm{kWh}$ and $0.37 € / \mathrm{kWh}$, respectively.

Initially, the planning problem is solved considering 14 days $(336 \mathrm{~h})$ in order to size the wind DG units and the ESSs in the candidate buses. The results (continuous sizes) of the OPF technique are presented in Table I. Next, the results of Table I are filtered and discrete sizes are given to the capacities of the wind DG units and the ESSs, as can be seen in Table II.

For the operation problem, two scenarios were considered. In the first scenario, ESSs are considered to operate under unity power factor, while in the second scenario, it is assumed that ESSs can contribute reactive power to the distribution network. The results of the economic analysis of the two scenarios are presented in Table III.

According to Table III, the ESSs' reactive power contribution seems that does not affect the annual revenue from the energy yield by the DG units and ESSs. On the contrary, a significant energy loss reduction is noticed in case that the ESSs contribute reactive power to the network. Consequently, the optimal operation strategy has to incorporate the ability of ESSs to contribute reactive power to the power system.

The active and reactive power of the system's substation before and after the installation of the DG units and the ESSs are presented in Figs. 1 and 2, respectively. It can be seen from Fig. 1 that when the wind power is high and the load demand is covered, active power is exported to the transmission system. This mostly happens during the peak days as well as the day with minimum load and peak wind speed. In order to satisfy all the system constraints, in these days a rather small percentage of wind power is curtailed, as can be seen in Fig. 3.

TABLE I

Continous SizES Of THE WIND DG UNITS AND ESSS

\begin{tabular}{|c|c|c|c|}
\hline Bus & $\mathbf{P}_{\text {wdg }}(\mathbf{M W})$ & $\mathbf{S}_{\max }(\mathbf{M W})$ & $\mathbf{E}_{\text {ess }}(\mathbf{M W h})$ \\
\hline 3 & 0.0000 & 0.2110 & 0.4045 \\
\hline 5 & 0.2104 & 0.0000 & 0.0000 \\
\hline 14 & 0.2038 & 0.0000 & 0.0000 \\
\hline 19 & 0.0000 & 1.5513 & 3.6923 \\
\hline 21 & 3.6765 & 0.0000 & 0.0000 \\
\hline 31 & 2.7451 & 0.5391 & 0.9380 \\
\hline
\end{tabular}


TABLE II

DisCRETE SIZES OF THE WIND DG UNITS AND ESSS

\begin{tabular}{|c|c|c|c|}
\hline Bus & $\mathbf{P}_{\text {wdg }}(\mathbf{M W})$ & $\mathbf{S}_{\max }(\mathbf{M W})$ & $\mathbf{E}_{\text {ess }}(\mathbf{M W h})$ \\
\hline 3 & 0.00 & 0.25 & 0.50 \\
\hline 5 & 0.50 & 0.00 & 0.00 \\
\hline 14 & 0.50 & 0.00 & 0.00 \\
\hline 19 & 0.00 & 1.90 & 3.80 \\
\hline 21 & 3.75 & 0.00 & 0.00 \\
\hline 31 & 2.75 & 0.50 & 1.00 \\
\hline
\end{tabular}

TABLE III

ECONOMic ANALysis Results For THE ANNUAL OPERATION OF THE INSTALLED DG UNITS AND THE ESSS

\begin{tabular}{|l|r|r|c|}
\hline \multicolumn{1}{|c|}{ Parameter } & OPF (€) & \multicolumn{1}{|c|}{$\begin{array}{c}\text { A-R OPF } \\
(\boldsymbol{\epsilon})\end{array}$} & $\begin{array}{c}\text { Difference } \\
(\boldsymbol{\%})\end{array}$ \\
\hline $\begin{array}{l}\text { Annual revenue from } \\
\text { energy production }\end{array}$ & 1168550 & 1168350 & 0.017 \\
\hline $\begin{array}{l}\text { Annual cost of energy } \\
\text { losses }\end{array}$ & 21804 & 18238 & -16.36 \\
\hline
\end{tabular}

Fig. 4 demonstrates how the PCS functions as a capacitor when it supplies reactive power (positive value) and as an inductor when it absorbs reactive power (negative value) in compliance with the needs of the system in order to eliminate the reactive power exchange. Fig. 5 shows the voltage magnitude of Bus 27 during the 14 representative days before and after the installation of the DG units and the ESSs. It can be seen that the ESSs' reactive power contribution improves the voltage profile of the bus.

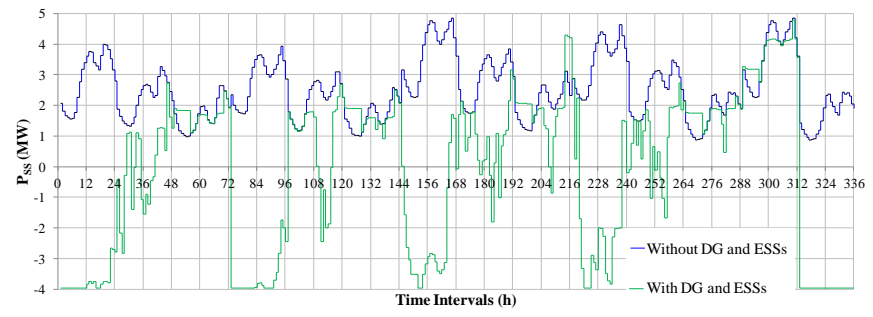

Fig. 1. Active power of the substation.

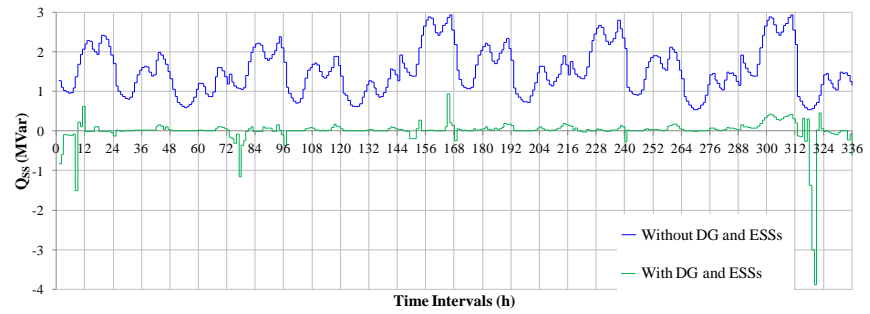

Fig. 2. Reactive power of the substation.

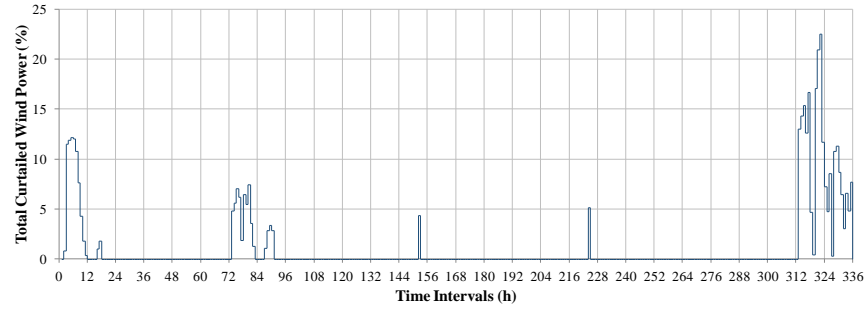

Fig. 3. Total percentage of the curtailed wind power.

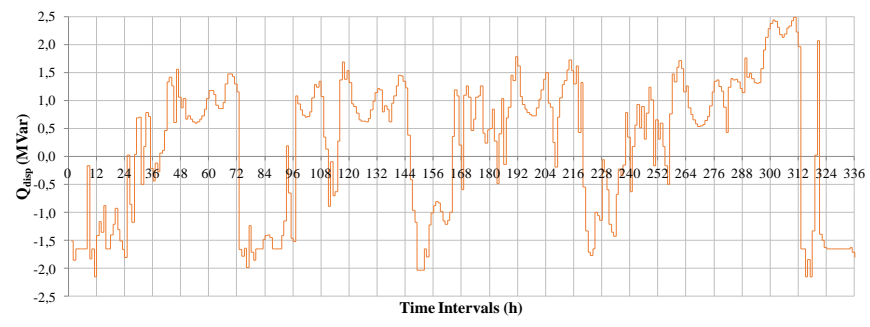

Fig. 4. Total reactive power of the ESSs.

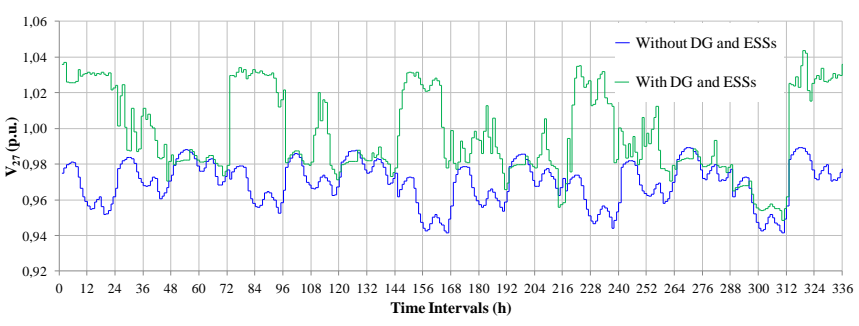

Fig. 5. Voltage profile of Bus 27.

\section{CONCLUSION}

This paper develops a method for the optimal sizing of wind power generation units and ESSs in a power distribution network and their optimal operation in order to maximize the revenues of the distribution network operator with consideration of the system's energy losses, in case that the distribution network operator is also the owner of DG units. A two-stage optimization scheme, comprimising an OPF-based method, is proposed. In the first stage, the optimal sizes of wind DG units and ESSs are obtained, while in the second stage an optimal operation strategy is derived considering the ESSs' reactive power contribution and a curtailment index of wind power generation. To demonstrate the effectiveness of the proposed approach, a 34-bus distribution system is used as a case study. It is shown that the usage of ESSs increases the penetration levels of wind energy, contributes to the annual energy losses reduction and improves the voltage profile of the system.

\section{ACKNOWLEDGEMENTS}

This work has been performed within the Operational Program "Education and Lifelong Learning", Project "Archimidis III", research project entitled "Design and 
development of an on-line smart power management system for Crete autonomous power system with very high penetration of renewable energy sources", which is co-financed by the European Union and the Greek Government.

\section{REFERENCES}

[1] Y. M. Atwa, E. F. El-Saadany, M. M. A. Salama and R. Seethapathy, "Optimal renewable resources mix for distribution system energy loss minimization," IEEE Trans. Power Syst., vol. 25, no. 1, Feb. 2010.

[2] A. Gabash and P. Li, "Active-Reactive optimal power flow for low voltage networks with photovoltaic distributed generation," in Proc. 2nd IEEE Int. Energy Conf. Exhib. (EnergyCon2012)/ Future Energy Grids and Systems (FEGS), Florence, Italy, Sep. 2012, pp. 381-386.

[3] The EU Climate and Energy Package, [Online]. Available at: http://ec.europa.eu/clima/policies/package/index_en.htm

[4] M. J. N. van Werven and M. J. J. Scheepers, "The changing role of energy suppliers and distribution system operators in the deployment of distributed generation in liberalized electricity markets, DISPOWER Rep. ECN-C-05-058, 2005.

[5] P. Poonpun and W. T. Jewell, "Analysis of the cost per kilowatt hour to store electricity," IEEE Trans. Power Syst., Vol. 23, No.2, pp. 529-534, Jun. 2008.
[6] A. Gabash and P. Li, "Active-reactive optimal power flow in distribution networks with embedded generation and battery storage," IEEE Trans. Power Syst., vol. 27, no. 4, pp. 2026-2035, Nov. 2012.

[7] Y. M. Atwa and E. F. El-Saadany, "Optimal allocation of ESS in distribution systems with a high penetration of wind energy," IEEE Trans. Power Syst., vol. 25, no. 4, pp. 1815-1822, Nov. 2010.

[8] GAMS - A User's Guide. New York, 2008

[9] A. Gabash and P. Li, "Flexible optimal operation of battery storage systems for energy supply networks," IEEE Trans. Power Syst., Sept. 2012.

[10] P. M. DeOliveira-DeJesus, M. T. PoncedeLeao, J. M. Yusta, H. M Khodr and A. J. Urdaneta, "Uniform marginal pricing for the remuneration of distribution networks" IEEE Trans. Power Syst., vol. 20, no. 3, pp. 1302-1310, Aug. 2005.

[11] N. W. Miller, R. S. Zrebiec, G. Hunt and R. W. Deimerico, "Design and commissioning of a 5 MVA, 2.5 MWh battery energy storage system," in Proc. IEEE Transm. Distrib. Conf., Los Angeles, CA, Aug. 2007, pp.339-345.

[12] GE Energy Storage Datasheet. Link: http://www.geenergystorage.com.

[13] RETScreen. Link: http://www.retscreen.net/el/home.php.

[14] Y. A. Katsigiannis, P. S. Georgilakis, and E. S. Karapidakis, "Hybrid simulated annealing-tabu search method for optimal sizing of autonomous power systems with renewables," IEEE Trans. Sustain. Energy, vol. 27, no. 4, pp. 2026-2035, Nov. 2012.

[15] M. Chris, M. M. A. Salama, and S. Jayaram, "Capacitor placement in distribution systems using heuristic strategies," IEE Proc. Gener. Transm. Distrib., vol. 144, no. 3, pp. 225-230, May 1997. 